\title{
Tumor genotype dictates radiosensitization after Atm deletion in primary brainstem glioma models
}

\author{
Katherine Deland, ${ }^{1}$ Bryce F. Starr, ${ }^{1}$ Joshua S. Mercer, ${ }^{1}$ Jovita Byemerwa, ${ }^{2}$ Donna M. Crabtree, ${ }^{3}$ Nerissa T. Williams, ${ }^{1}$ Lixia Luo, ${ }^{1}$ \\ Yan Ma, ${ }^{1}$ Mark Chen, ${ }^{2,4}$ Oren J. Becher, ${ }^{5,6,7}$ and David G. Kirsch ${ }^{1,2}$ \\ 'Department of Radiation Oncology, ${ }^{2}$ Department of Pharmacology \& Cancer Biology, ${ }^{3}$ Department of Pediatrics, and ${ }^{4}$ Medical Scientist Training Program, Duke University Medical Center, Durham, \\ North Carolina, USA. ${ }^{5}$ Department of Pediatrics and ${ }^{6}$ Department of Biochemistry and Molecular Cenetics, Northwestern University, Chicago, Illinois, USA. ${ }^{7}$ Ann and Robert H. Lurie Children's Hospital \\ of Chicago, Chicago, Illinois, USA.
}

\begin{abstract}
Diffuse intrinsic pontine glioma (DIPC) kills more children than any other type of brain tumor. Despite clinical trials testing many chemotherapeutic agents, palliative radiotherapy remains the standard treatment. Here, we utilized Cre/loxP technology to show that deleting Ataxia telangiectasia mutated (Atm) in primary mouse models of DIPC can enhance tumor radiosensitivity. Genetic deletion of Atm improved survival of mice with $p 53$-deficient but not $p 53$ wild-type gliomas after radiotherapy. Similar to patients with DIPG, mice with $p 53$ wild-type tumors had improved survival after radiotherapy independent of Atm deletion. Primary $p 53$ wild-type tumor cell lines induced proapoptotic genes after radiation and repressed the NRF2 target, NAD(P)H quinone dehydrogenase 1 (Nqo1). Tumors lacking $p 53$ and Ink4a/Arf expressed the highest level of Nqo1 and were most resistant to radiation, but deletion of $A t m$ enhanced the radiation response. These results suggest that tumor genotype may determine whether inhibition of ATM during radiotherapy will be an effective clinical approach to treat DIPGs.
\end{abstract}

\section{Introduction}

Diffuse intrinsic pontine glioma (DIPG), also referred to as highgrade brainstem glioma, is an incurable cancer that originates in the pons and occurs primarily in children. Focal radiation therapy is the current standard of care for children with DIPG because the anatomical location of the tumor and its infiltrating growth patterns preclude surgery. Furthermore, no chemotherapeutic agents have improved outcome over radiotherapy alone in clinical trials. Although neurological symptoms typically improve after radiotherapy, the tumors invariably recur. The median survival for children with DIPG is less than 1 year, and fewer than $10 \%$ of patients survive 2 years after diagnosis $(1,2)$. Therefore, DIPG is the major cause of brain cancer-related deaths in children (1). One approach to improve the survival of children with DIPG involves developing effective strategies to overcome the radiation resistance of brainstem gliomas.

Given that damage to DNA is the primary cause of cell death by ionizing radiation (3), the proteins that sense DNA damage and signal the cell to repair damaged DNA play an essential role in maintaining genomic stability and regulating radiation response. Therefore, small-molecule drugs that interfere with the DNA damage response represent a promising strategy for enhancing the ability of radiotherapy to kill cancer cells (4). Furthermore, a

Conflict of interest: DGK is a cofounder of and receives research support from XRad Therapeutics, which develops radiosensitizers. DGK is on the scientific advisory board of and owns stock in Lumicell Inc., which develops intraoperative imaging technology. DCK receives research support from Merck, Bristol Myers Squibb, and XRad Therapeutics. Copyright: (c) 2021, American Society for Clinical Investigation.

Submitted: July 10, 2020; Accepted: September 23, 2020; Published: January 4, 2021.

Reference information: J Clin Invest. 2021;131(1):e142158.

https://doi.org/10.1172/JCl142158. recent RNAi screen in patient-derived DIPG cells identified several kinases involved in the DNA-damage response whose inhibition selectively impaired survival after radiation exposure (5). One such kinase, ataxia telangiectasia mutated (ATM) is recruited to sites of damage and activated through autophosphorylation (6-8). ATM phosphorylates numerous downstream effector proteins to orchestrate DNA repair (e.g., p53, MRE11, RAD50, BRCA1) (9) and contributes indirectly to DNA repair by phosphorylating proteins that facilitate cell cycle arrest and chromatin relaxation (e.g., CHK2, KAP1) (10-13).

Consistent with the role of ATM as a critical regulator of the DNA damage response, patients with ataxia telangiectasia who have 2 germline mutations in ATM are hypersensitive to radiation $(14,15)$. Similarly, Atm $^{-/}$mice die when exposed to sublethal radiation doses (16). Therefore, pharmacological inhibition of ATM kinase represents a rational approach to radiosensitize human tumor cells $(17,18)$. Importantly, the development of an ATM inhibitor that penetrates the blood-brain barrier (19) has enabled a phase I clinical trial of adult patients with glioblastoma that is testing concurrent ATM inhibition and radiotherapy (ClinicalTrials.gov NCT03423628). If this clinical trial shows that ATM inhibition during radiotherapy is safe, then testing this approach in DIPG patients could follow if preclinical studies demonstrate that targeting ATM improves survival in preclinical models of DIPG.

Here, we utilized primary genetically engineered mouse models (GEMMs) of brainstem glioma with different genotypes to explore the impact of tumor genotype and Atm deletion on tumor response to radiotherapy. We found that $p 53$-deficient but not $p 53$ wild-type brainstem gliomas (driven by deletion of Ink $4 a$ ) Arf) were radiosensitized by disruption of ATM signaling. Consistent with data from human DIPG patients (5), mice with $p 53$ wild-type brainstem gliomas exhibited an enhanced radiation 
response. The inherent radiosensitivity of these Ink4a/Arf-deficient tumors was mediated by $\mathrm{p} 53$ signaling and associated with repression of the NRF2 pathway gene NAD(P)H quinone dehydrogenase 1 (Nqo1). We showed that deletion of $p 53$ in the Ink4a/ Arf-deficient gliomas resulted in overexpression of $\mathrm{NqO1}$ and significantly increased radiation resistance in vivo. Importantly, deletion of Atm in the radioresistant brainstem gliomas lacking p53 improved the survival of tumor-bearing mice treated with radiotherapy. Our findings suggest that ATM is a promising target for the radiosensitization of DIPGs and that the outcomes of clinical trials testing concurrent ATM inhibition and radiotherapy should be stratified according to $p 53$ mutational status.

\section{Results}

Generating primary brainstem gliomas. To investigate whether disruption of Atm can modulate the radiosensitivity of DIPGs, we employed Cre-loxP and replication-competent avian sarcomaleukosis virus long-terminal repeat with splice acceptor-tumor virus A (RCAS-TVA) technology to generate primary brainstem gliomas as previously described (20). Chicken fibroblast cells producing various RCAS retroviruses were injected into the brainstem of neonatal Nestin ${ }^{T V A}$ mice, in which Nestin-expressing progenitor cells expressed the RCAS cognate TVA receptor (Figure 1A). We initiated glioma development by using RCAS-PDGF subunit B (RCAS-PDGFB) to overexpress the PDGFB oncogene and RCASCre to delete floxed alleles of various tumor suppressors (Figure 1B). In addition to promoting tumorigenesis, Cre recombinase also deleted either 1 or 2 floxed alleles of Atm in order to modulate the radiosensitivity of tumor parenchymal cells.

Injection of mice with chicken fibroblasts expressing RCASluciferase enabled tumor development to be confirmed in vivo via bioluminescence (Figure 1A). To verify that bioluminescence in the brain was indicative of a tumor, we performed MRI on a subset of mice with detectable luciferase activity. Mice with bioluminescence in the brain 4.5 weeks after injection had well-defined tumors within the brainstem visualized by MRI (Figure 1, C and D). The tumors enhanced after i.v. injection of gadolinium contrast, indicating that the blood-brain barrier was compromised (Figure 1, C and D). Furthermore, the overexpression of PDGFB was confirmed in primary gliomas by immunohistochemistry for the HA tag on PDGFB (Figure 1, E and F).

Deletion of Atm radiosensitizes p53-deficient brainstem gliomas. Exome sequencing of DIPG samples from patients has revealed that mutations in the tumor suppressor $p 53$ occur in $50 \%-77 \%$ of tumors. Likewise, amplification of PDGF receptor $\alpha$ (PDGFRA) is a common molecular alteration, found in $20 \%-40 \%$ of DIPGs $(21,22)$. To model PDGF-driven, p53-deficient brainstem gliomas, we initiated tumor development in Nestin ${ }^{T V A} p 53^{f l f l} \mathrm{Atm}^{f l++}$ $\left(n P A^{f l /+}\right)$ and Nestin ${ }^{T V A} p 53^{f / f l} A_{t m^{f l / f l}}\left(n P A^{f l / f l}\right)$ mice. $n P A^{f l /+}$ mice retained expression of 1 wild-type allele of Atm after Cre-mediated recombination and served as the control for all experiments, whereas $n P A^{f l / f l}$ mice lacked ATM function. Cre-mediated recombination of floxed alleles of $p 53$ and Atm was confirmed by performing droplet digital PCR on genomic DNA from primary glioma cells sorted by flow cytometry (Figure 2A and Supplemental Figure 1; supplemental material available online with this article; https://doi.org/10.1172/JCI142158DS1).
A recent study reported that knocking out ATM in tumor cells decreased colony formation in soft agar and slowed tumor growth in immunocompromised mice (23). In a primary brainstem glioma model, however, we detected no difference in the time to tumor detection by in vivo bioluminescence imaging between the Atm-expressing and Atm-deficient gliomas (Figure 2B). Likewise, deletion of Atm in the glioma cells did not affect overall survival of tumor-bearing mice in the absence of radiotherapy (Figure 2C).

To characterize ATM signaling in these brainstem gliomas, we performed immunohistochemistry after radiation therapy. The number of cells staining positively for the activated and phosphorylated form of ATM (pATM) or the ATM target, KRAB-associated protein-1 (pKAP1), was significantly diminished after 10 Gy in $n P A^{f l f l}$ gliomas when compared with $n P A^{f l++}$ gliomas (Figure 2, D and E, and Supplemental Figure 2). These data provide independent validation of Atm recombination in brainstem glioma cells in vivo, which resulted in disruption of ATM signaling after radiation therapy.

p53-deficient glioma cells lacking both Atm alleles displayed heightened radiosensitivity in vivo and in vitro. The disruption of ATM signaling resulted in enhanced tumor cell death 24 hours after 10 Gy whole-brain irradiation, as detected by TUNEL staining (Figure $2 \mathrm{~F}$ and Supplemental Figure 2). Similarly, early passage cell lines from tumors in $n P A^{f / f l}$ mice were significantly more sensitive to radiation than cell lines derived from $n P A^{f /+}$ mice in clonogenic survival assays in vitro (Figure 2G). To investigate whether enhancing tumor cell radiosensitivity improves the survival of mice with primary gliomas treated with radiotherapy, we irradiated $n P A^{f l++}$ and $n P A^{f l / f}$ mice with 3 daily fractions of 10 Gy to the brain. The elevated radiosensitivity of glioma cells in $n P A^{f / f f}$ mice translated to a tripling of the median survival after radiation therapy (Figure $2 \mathrm{H}$ ). These data indicate that targeting ATM represents a promising therapeutic approach to improve the response of brainstem gliomas to radiation therapy.

Deletion of Atm in 553 wild-type gliomas does not improve radiation response. Biddlestone-Thorpe et al. reported that mutations in $p 53$ were required for radiosensitization by ATM inhibition in a xenograft model of glioblastoma (24). To assess whether loss of p53 signaling was also required for radiosensitization of primary gliomas lacking Atm expression, we generated $p 53$ wild-type tumors. Overexpression of PDGFB without simultaneous deletion of a tumor suppressor resulted in the formation of indolent, lowgrade gliomas with a low mitotic index (25). In order to efficiently model high-grade gliomas that retained expression of $p 53$ and had a similar latency as $p 53$-deficient tumors, we therefore combined $P D G F B$ overexpression with recombination of both alleles of the tumor suppressor Ink4a/Arf. Tumor development was initiated in Nestin ${ }^{T V A}$ Ink4a/Arfl/fl Atm $^{f l /+}\left(n I A^{f /+}\right)$ and Nestin ${ }^{\text {TVA }}$ Ink4a/Arfl/fl $A_{t m}^{f l f l}\left(n I A^{f l / f l}\right)$ mice. Although p19 ${ }^{\text {ARF }}$, an alternate reading frame product from the Ink4a/Arf locus, functioned to stabilize p53 in response to oncogenic stress, Ink4a/Arf-deficient tumor cells in this model retained the ability to induce and activate $\mathrm{p} 53$ in response to radiation (Supplemental Figure 3). After verifying that the Ink4a/ Arf-deficient cells were functionally p53 intact, we quantified the recombination efficiency of floxed Atm and Ink4a/Arf alleles using droplet digital PCR. Most flow-sorted primary glioma cells demonstrated full recombination of the floxed Ink $4 a / A r f$ alleles 

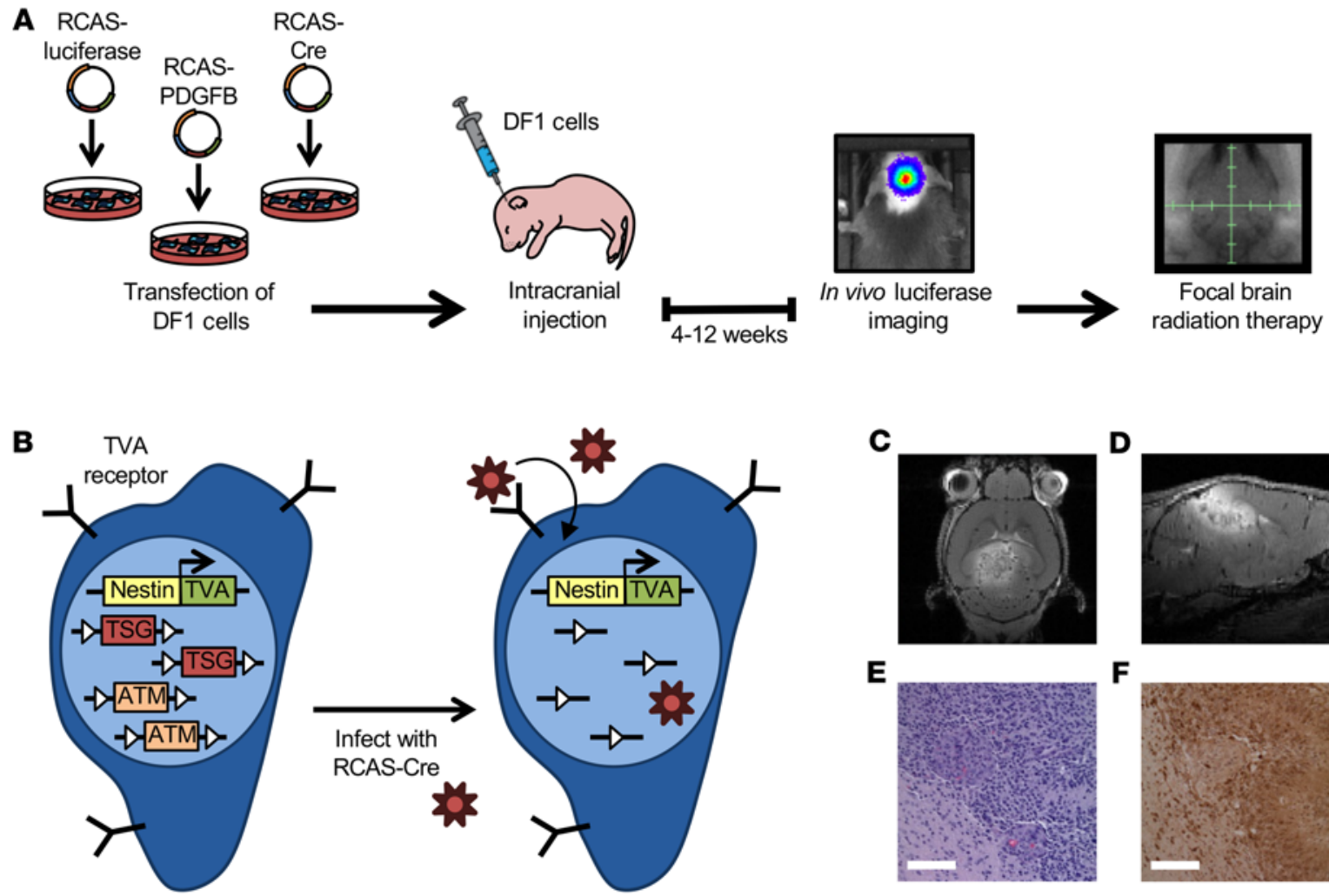
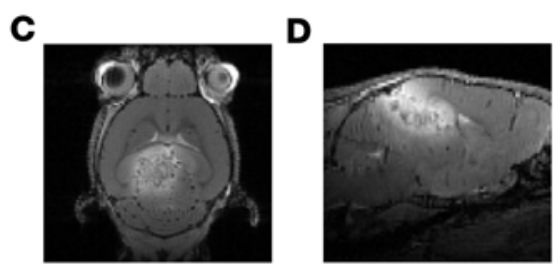

$\mathbf{E}$

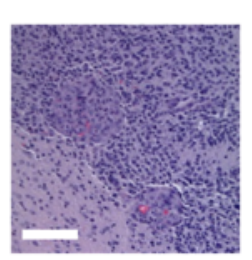

$\mathbf{F}$

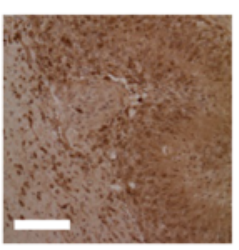

Figure 1. Genetically engineered mouse model of brainstem glioma. (A) DF1 chicken fibroblast cells were transfected with RCAS constructs expressing luciferase, PDGFB, or Cre. The virus-producing DF1 cells were injected into the brainstem of neonatal mice. Mice were subjected to biweekly bioluminescence imaging to confirm the presence of a brainstem glioma. Upon tumor detection, mice were stratified to various treatment cohorts. (B) Schematic showing RCAS-Cre-mediated recombination of floxed alleles of Atm and tumor suppressor genes (TSG) in Nestin-expressing neural progenitor cells that harbor the TVA receptor. Triangles represent loxP sites. (C) Axial and (D) sagittal plane views of the glioma enhanced with gadolinium contrast by MRI. Representative glioma stained with (E) H\&E or (F) an antibody recognizing the HA tag on PDCFB. Scale bars: $100 \mu \mathrm{m}$.

(Supplemental Figure 4), and there was similar recombination efficiency of Atm in the tumors from $n I A^{f /+}$ and $n I A^{f / f l}$ mice (Figure $3 \mathrm{~A}$ ).

In order to evaluate the impact of Atm deletion on growth of $p 53$ wild-type gliomas, we characterized primary tumor development in $n I A^{f /+}$ and $n I A^{f l / f l}$ mice. Consistent with data generated in the p53-deficient model, deletion of Atm did not significantly alter time to tumor detection by in vivo imaging (Figure $3 \mathrm{~B}$ ) or the overall survival of tumor-bearing mice in the absence of radiation (Figure $3 \mathrm{C}$ ).

We also utilized immunohistochemistry to characterize ATM signaling in primary $p 53$ wild-type tumors after radiation. Staining for pATM and the downstream target pKAP1 was reduced significantly after 10 Gy radiation exposure in $n I A^{f / f l}$ gliomas compared with $n I A^{f l /+}$ gliomas that retained Atm expression (Figure 3, D and E and Supplemental Figure 5). Although phosphorylated p53 was detected in both genotypes, the deletion of Atm significantly decreased the activation of p53 in response to radiation (Supplemental Figure 6). Despite the significant disruption of ATM signaling after radiation in $n I A^{f / f l}$ gliomas, we did not detect an increase in radiation-induced cell death by TUNEL staining (Figure 3F). Furthermore, deletion of Atm in p53 wild-type gliomas did not translate to improved survival after radiotherapy in vivo (Figure $3 G$ ). Therefore, lack of functional ATM regulated the response of primary brainstem gliomas to radiation therapy in $p 53$-deficient but not Ink4a/Arf-deficient ( $p 53$ wild-type) tumors. p53 wild-type brainstem gliomas display enhanced radiosensitivity. A recent study using patient-derived DIPG cells and a retrospective cohort of DIPG patients revealed that mutations in $p 53$ drive radioresistance (5). Most of the patients with p53 wild-type DIPGs in this study were classified as good clinical and radiological responders after radiation therapy, whereas only a minority of $p 53$ mutant tumors could be classified similarly. To determine whether $p 53$ status influences radiation resistance in the primary models of brainstem glioma, we compared the radiation response of $p 53-$ deficient and wild-type gliomas in $\mathrm{Atm}^{\mathrm{fl} /+}$ mice that retained Atm expression. Although survival of mice with $p 53$-deficient tumors tripled in response to radiotherapy (27-day increase in median survival), radiotherapy resulted in a 12-fold increase in the survival of mice bearing $p 53$ wild-type tumors (110-day increase in median survival) (Figure 4, A and B). Importantly, primary gliomas driven by loss of either $p 53$ or Ink4a/Arf grew at a similar rate in the absence of radiation therapy (Figure 4C). However, after 3 daily fractions of wholebrain irradiation, mice with $p 53$ wild-type tumors survived longer than mice with $p 53$-deficient tumors (Figure 4D). The survival of mice bearing $p 53$ wild-type gliomas was comparable to the survival of $n P A^{f / f l}$ mice with tumors lacking Atm. Collectively, these data suggest that $p 53$ wild-type tumors driven by loss of Ink $4 a /$ Arf had enhanced sensitivity to radiation therapy (Table 1). These radiosensitive brainstem gliomas could not be further radiosensitized by loss of Atm. 

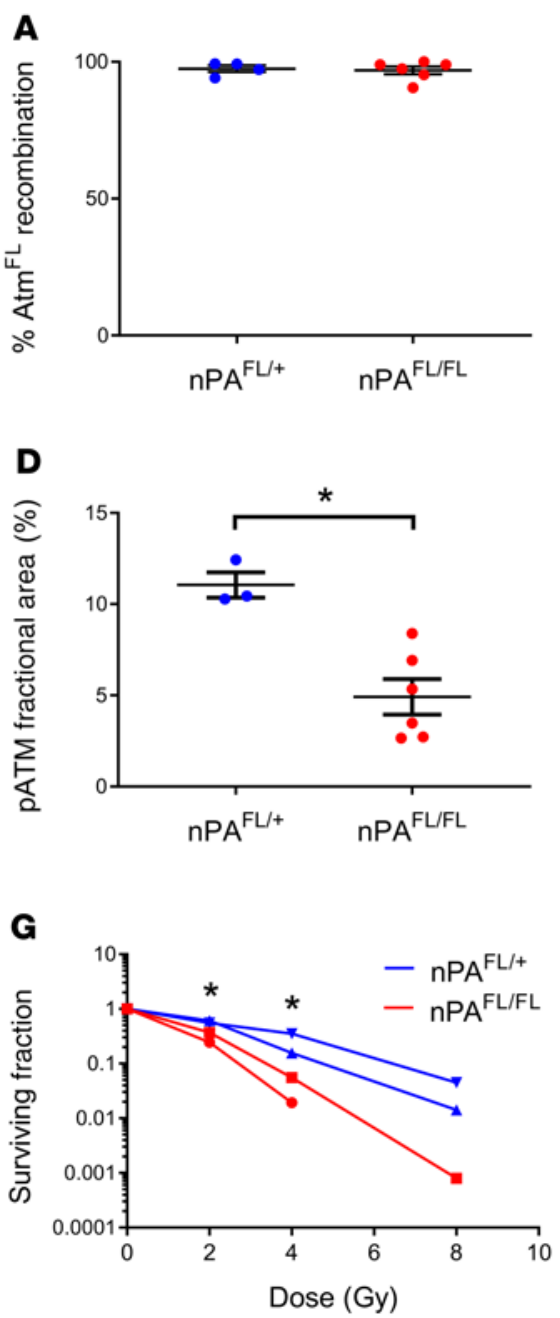
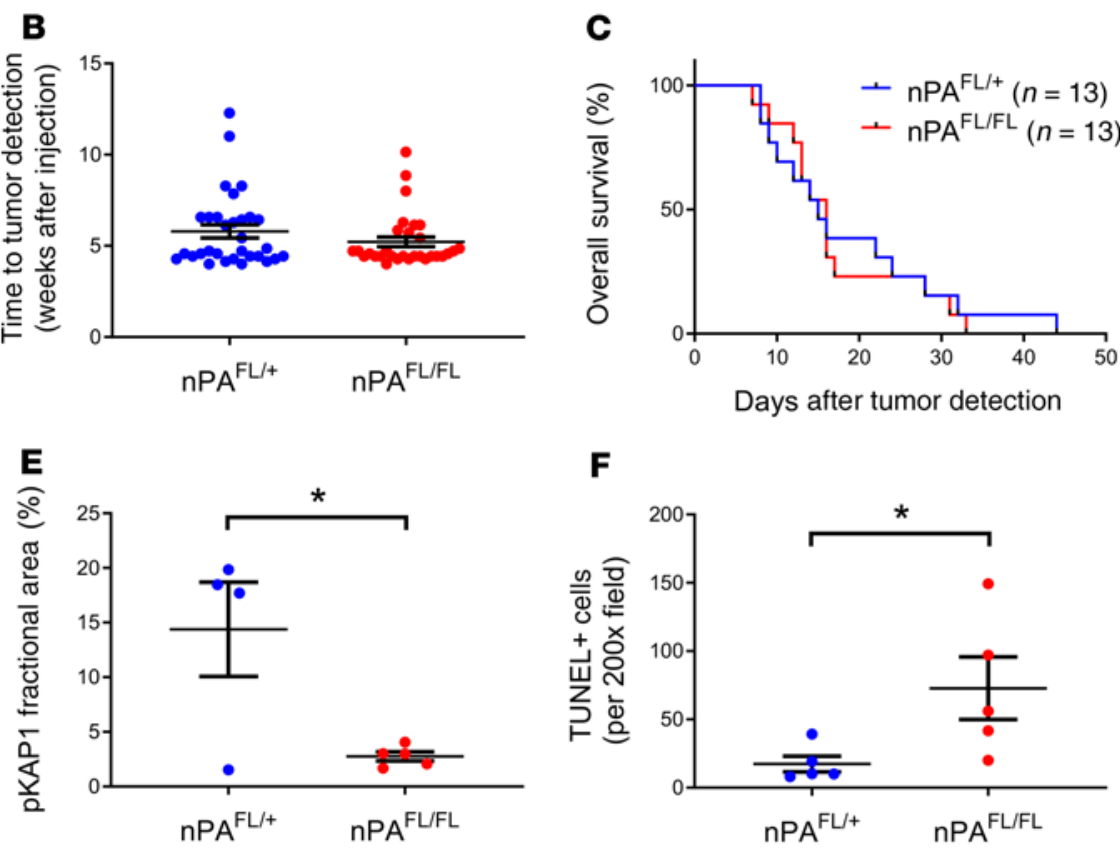

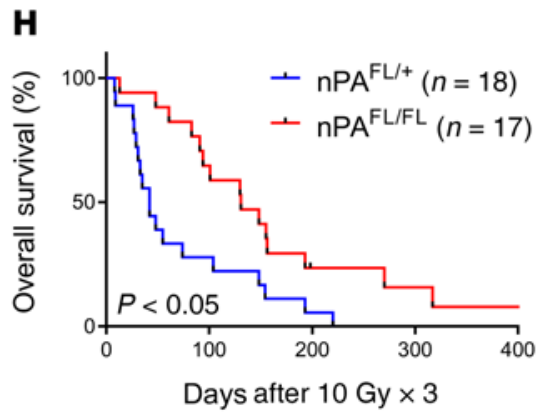

Figure 2. Deletion of Atm in p53-deficient gliomas improves tumor response to radiation. (A) Percentage recombination of the floxed allele of $A t m$ in flow-sorted glioma cells as assessed by droplet digital PCR. (B) Time to tumor development after injection in $n P A^{f / /+}$ and $n P A^{f / f l}$ mice as detected through in vivo bioluminescence imaging. Each circle represents 1 mouse. (C) Kaplan-Meier plot of overall survival in tumor-bearing mice after tumor detection. Quantification of the fractional area of positive staining for (D) pATM 2 hours after 10 Gy or (E) pKAP1 1 hour after 10 Gy in primary gliomas. (F) Number of TUNEL ${ }^{+}$cells in $n P A^{f / /+}$ and $n P A^{f / / f l}$ tumors 24 hours after 10 Gy whole-brain irradiation. ${ }^{*} P<0.05$ by $t$ test ( 2 tailed). (G) Clonogenic survival assay using stroma-depleted $n P A^{f / /+}$ and $n P A^{f l / f l}$ tumor cell lines ( $n=2$ cell lines per genotype). ${ }^{*} P<0.05$ by 2 -way ANOVA followed by Bonferroni's multiple-comparison test. (H) Kaplan-Meier plot of overall survival in tumor-bearing mice after 3 daily fractions of 10 Gy radiation therapy delivered to the whole brain. ${ }^{*} P<0.05$ by log rank test.

Proapoptotic genes are induced after radiation in $p 53$ wild-type gliomas. We hypothesized that the radiosensitivity of Ink4a/ Arf-deficient tumors may be caused by the transactivation of p53 target genes in response to radiation. To evaluate whether genes associated with $\mathrm{p} 53$-mediated cell death are induced in primary glioma cells, we quantified the expression of Bax, Puma, and Noxa using real-time PCR. These proapoptotic genes were induced 4 hours after radiation exclusively in the Ink $4 a /$ Arf-deficient cells (Figure 5, A-C). In order to confirm that the radiation-induced expression of these proapoptotic genes was driven by $\mathrm{p} 53$, we generated tumors lacking both $p 53$ and Ink4a/Arf in Nestin ${ }^{T V A} p 53^{f / f l}$ Ink $4 a / A r f^{f l / f l} A^{t m}{ }^{f l++}\left(n P I A^{f l /+}\right)$ mice. Deletion of $p 53$ in the Ink4a/ Arf-deficient gliomas prevented the induction of Bax, Puma, and Noxa after radiation (Figure 5, A-C). Similarly, an increase in cleaved caspase-3 after irradiation was observed in p53 wildtype glioma cells compared with cells lacking both $p 53$ and Ink4a/ Arf (Figure 5D). These data suggest that the radiosensitivity of p53 wild-type brainstem gliomas may be mediated in part by the p53-dependent accumulation of proapoptotic proteins.

Brainstem gliomas lacking both $p 53$ and Ink4a/Arf are resistant to radiation. To test whether the transactivation of $\mathrm{p} 53$ target genes was responsible for the enhanced radiosensitivity of Ink4a/Arfdeficient brainstem gliomas, we followed tumor-bearing $n P I A^{f /+}$ mice for survival with and without radiation exposure. The deletion of $p 53$ in the Ink4a/Arf-deficient gliomas did not accelerate tumor growth in the absence of radiation (Figure 5E). However, the disruption of p53 signaling after radiation attenuated the radiosensitivity of Ink $4 a / A r f$-deficient tumors and significantly reduced the survival of tumor-bearing mice (Figure 5F). Surprisingly, the brainstem gliomas lacking both tumor suppressors were more resistant to radiation than the $p 53$-deficient gliomas, as indicated by a significant decrease in survival after fractionated radiotherapy (Figure $5 \mathrm{~F}$ and Table 1). These results suggest that $\mathrm{p} 53$ and INK4A/ARF independently contribute to cell survival in response to radiotherapy. 
A
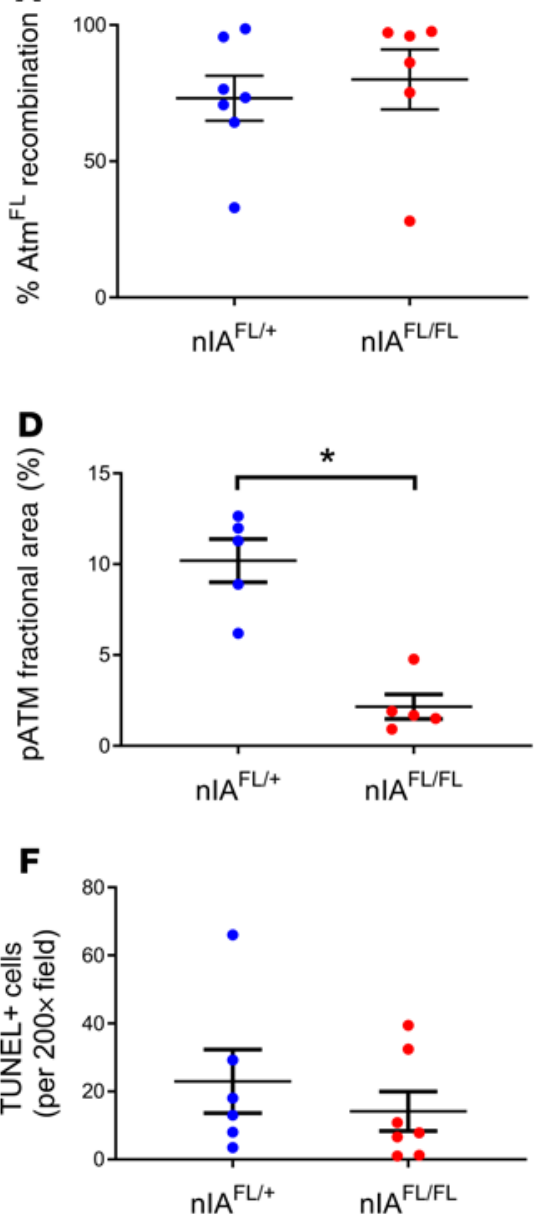

B
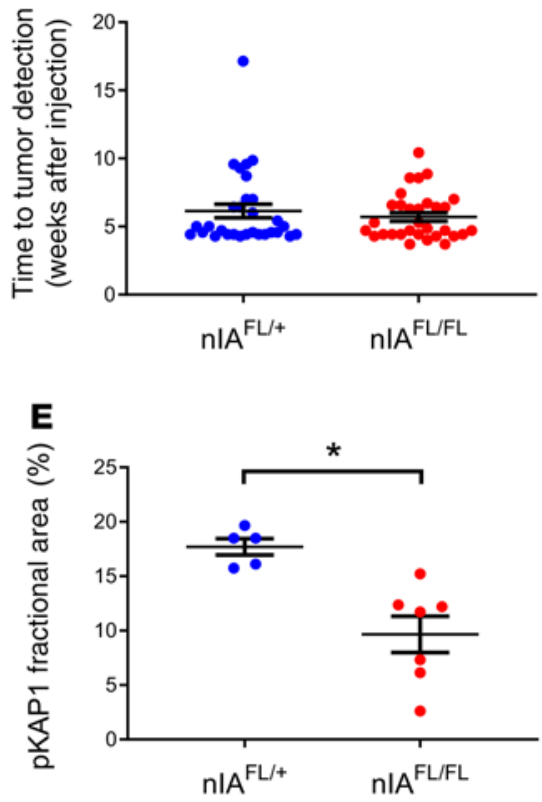

G

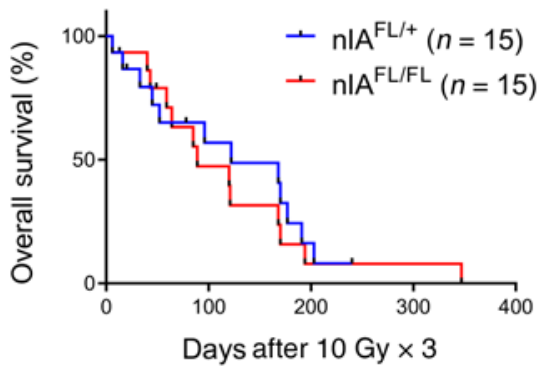

C

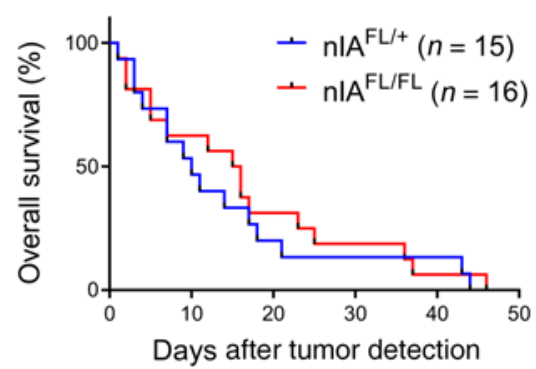

Figure 3. Deletion of Atm does not radiosensitize $p 53$ wild-type gliomas. (A) Percentage recombination of the floxed allele of Atm in flow-sorted glioma cells as assessed by droplet digital PCR. (B) Time to tumor development after injection in $n / A^{f / /+}$ and $n \mid A^{f l / f l}$ mice as detected through in vivo bioluminescence imaging. (C) Kaplan-Meier plot of overall survival in tumor-bearing mice after tumor detection in the absence of radiotherapy. Quantification of the fractional area of positive staining for (D) PATM 2 hours after 10 Gy or (E) pKAP1 1 hour after 10 Gy in primary brainstem gliomas. (F) Number of TUNEL ${ }^{+}$cells in $n / A^{f / /}$ and $n / A^{f / f l}$ tumors 24 hours after 10 Gy whole-brain irradiation. (C) Kaplan-Meier plot of overall survival in tumor-bearing mice after 3 daily fractions of 10 Gy radiation therapy delivered to the whole brain. ${ }^{*} P<0.05$ by $t$ test ( 2 tailed).
Dysregulation of the NRF2 pathway is an established mechanism of radiation resistance in human tumors and mouse models of cancer $(26,27)$. NRF2 acts as a master transcriptional regulator of the cellular response to oxidative stress and alters the response to radiation by reducing intracellular ROS and DNA damage $(26,28)$. Furthermore, a recent study found that NRF2 and its transcriptional target SLC7A11 are negatively regulated by p 53 and ARF through independent mechanisms (29). ARF directly interacts with NRF2 and prevents it from binding to the cognate antioxidant response element (ARE), and p53 appears to repress transcription of NRF2 targets by binding to ARE-containing promoters $(30,31)$. Therefore, we reasoned that the enhanced radioresistance of brainstem gliomas lacking both $p 53$ and Ink4a/Arf may be partially mediated by hyperactivation of NRF2 target genes (Figure $5 \mathrm{G}$ ).

To test this hypothesis, we utilized real-time PCR to examine the expression of the NRF2 target gene Nqo1. We found that p53-deficient glioma cells had elevated expression of Nqo1 compared with $p 53$ wild-type cells (Figure $5 \mathrm{H}$ ). Deletion of Ink4a/Arf in addition to $p 53$ further increased $\mathrm{Nqo1}$ expression, suggesting that both $\mathrm{p} 53$ and INK4A/ARF function to negatively regulate this NRF2 target. We also observed accumulation of NQO1 protein in $p 53$ and Ink4a/Arf-deficient glioma cells (Figure 5I). Importantly, the $p 53$ wild-type tumors lacked Nqo1 mRNA and protein, correlating with their enhanced radiosensitivity. Although many signaling pathways have been shown to promote radiation resistance $(4,32)$, these data suggest that hyperactivation of the NRF2 pathway is associated with the increased radioresistance of $n P A^{f /++}$ and $n P I A^{f l /+}$ brainstem gliomas.

Deletion of Atm reduces the radioresistance of gliomas lacking p53 and Ink4a/Arf. To determine whether targeting ATM can overcome the marked radioresistance of tumors lacking both p53 and Ink4a/Arf, we initiated tumorigenesis in $n P I A^{f l /+}$ and Nestin ${ }^{\text {TVA }}$ p53 $3^{f / f l}$ Ink4a/Arfl/fl Atm $^{f l / f l}$ (nPIA ${ }^{f l / f l}$ ) mice. Consistent with our previous results, deletion of Atm in primary brainstem gliomas did not affect the time to tumor formation (Figure 6A) or accelerate tumorigenesis after detection (Figure 6B). We assessed the recombination of floxed alleles of Atm by staining tumor tissue for pATM after irradiation with $10 \mathrm{~Gy}$. Activation of ATM was significantly decreased in $n P I A^{f l / f l}$ tumors compared with $n P I A^{f l /+}$ tumors, where expression of 1 wild-type allele of Atm was retained (Figure 6C and Supplemental Figure 7).

Despite effective disruption of ATM signaling in nPIA $A^{f / f l}$ mice, we did not observe an increase in cell death 24 hours after radiation exposure (Figure 6D and Supplemental Figure 7). Nevertheless, the deletion of Atm modestly improved the survival of tumor-bearing mice treated with fractionated radiotherapy (Figure 6E). These results further demonstrated that in primary brainstem gliomas, the status of $p 53$ was a critical determinant of tumor response to concurrent radiotherapy and inactivation of ATM. Interestingly, the survival after irradiation of $n P I A^{f / f l}$ mice with 

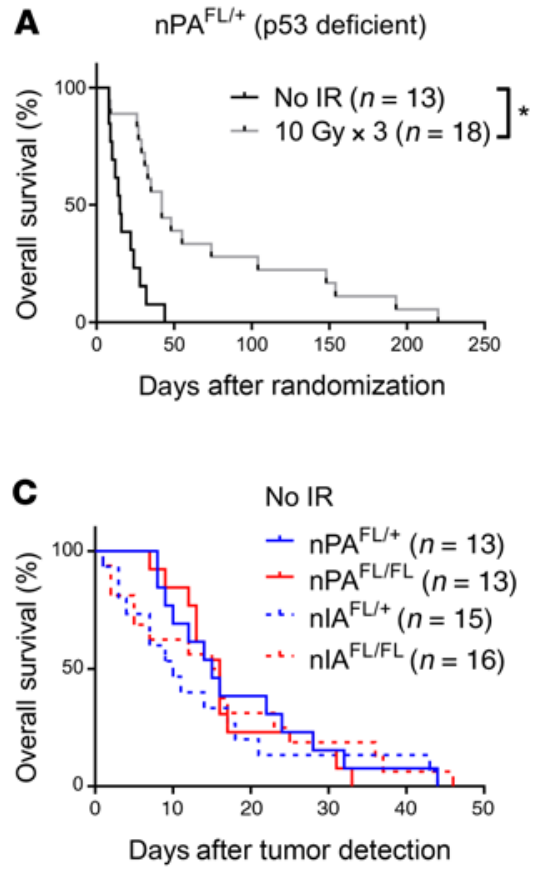
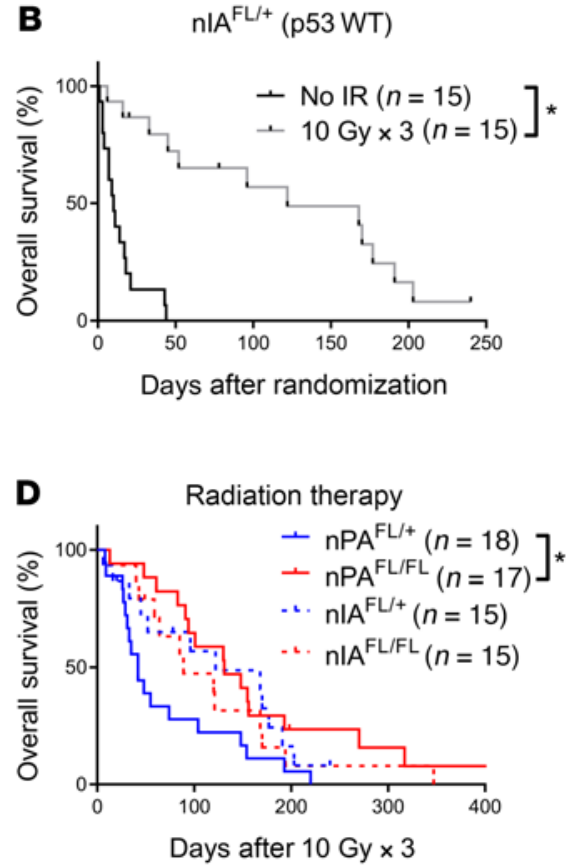

Figure 4. p53 wild-type gliomas with intact ATM function are sensitive to radiation. KaplanMeier plot of overall survival in (A) $p 53$-deficient tumor-bearing $n P A^{f l /+}$ mice or (B) $p 53$ wild-type tumor-bearing $n I A^{f /+}$ mice that received no radiotherapy or were treated with 3 daily fractions of 10 Gy to the whole brain. (C) Kaplan-Meier plot comparing the survival of unirradiated mice with p53 wild-type and p53-deficient brainstem gliomas. (D) Kaplan-Meier plot comparing the survival of mice with $p 53$ wild-type and $p 53$-deficient gliomas treated with fractionated radiotherapy. The animals included in these survival curves are the same animals from the survival studies in Figures 2 and $3 .{ }^{*} P<0.05$ by log rank test. tumors lacking both copies of $p 53$, Ink4a/Arf, and Atm was comparable to $n P A^{f l /+}$ mice whose tumors lacked $p 53$ but retained expression of Ink4a/Arf and Atm (Table 1), indicating that Atm-deficient gliomas driven by loss of $p 53$ and Ink4a/Arf remained relatively radioresistant (Figure 6F). Whereas deletion of Atm tripled the radiation response of $p 53$-deficient tumors that retained Ink4a/Arf expression, the same genetic modification only increased survival by 1.5 -fold in tumors lacking both $p 53$ and Ink4a/Arf (Table 1). Therefore, other genetic alterations could limit the ability of ATM inactivation to fully radiosensitize $p 53$-deficient gliomas.

\section{Discussion}

To efficiently characterize and modulate the radioresistance of DIPGs, we generated GEMMs of brainstem glioma by combining Cre-loxP and RCAS-TVA technology (20). These sophisticated techniques enabled precise control over the cell of origin and the genetic modifications driving gliomagenesis (33). Because genetic alterations in PDGFRA and the tumor suppressor $p 53$ are 2 of the most common genetic alterations in human DIPGs, we first investigated radiation response in a primary brainstem glioma model overexpressing $P D G F B$ and lacking $p 53$. We also generated a second model in which p53 function was retained but Ink4a/Arf was deleted in Nestin-expressing progenitor cells. Although mutations in the CDKN2A locus are rare in DIPGs, this locus is epigenetically repressed by the prevalent $\mathrm{K} 27 \mathrm{M}$ mutation in histone $\mathrm{H} 3.3$ (25). One limitation of this study is that we did not directly assess the impact of the H3.3K27M mutation on the radiation response of DIPGs or response to Atm deletion. However, it is noteworthy that a recent shRNA screen identified ATM as a promising target for the radiosensitization of H3.3K27M-mutated DIPG cells (5).

In addition to incorporating the genetic alterations found in many DIPG patients, primary models of brainstem glioma have been shown to resemble human tumors histologically and to undergo a similar pattern of invasion (34). Furthermore, by delivering fractionated radiotherapy alone to these mouse models, we were able to achieve a modest improvement in survival but not a tumor cure, which mimics the clinical response of DIPG patients treated with radiotherapy. Using the various GEMMs, we also showed that $p 53$ wild-type gliomas were more radiosensitive than $p 53$-deficient gliomas, in agreement with a retrospective analysis of DIPG patients treated with radiotherapy (5). Given that our primary tumor models closely phenocopy human brainstem gliomas, these murine models represent valuable tools for identifying drug targets that may improve clinical outcomes in DIPG patients treated with radiation therapy.

Utilizing complementary p53-deficient and p53 wild-type (Ink4a/Arf-deficient) models of brainstem glioma, we found that disruption of ATM signaling preferentially radiosensitized tumors lacking $p 53$. Although other groups have reported that $p 53$ status regulates radiosensitization by ATM inhibition $(24,35)$, our study is the first to our knowledge to implicate $p 53$ status in radiosensitization by Atm deletion in a primary tumor system. Furthermore, we previously reported that endothelial cell-specific deletion of Atm is not sufficient for radiosensitization after focal heart irradiation, but simultaneous loss of $p 53$ and Atm facilitates radiation-induced heart injury (36). Collectively, this body of work suggests that in tumors at specific anatomical sites, targeting ATM may provide a therapeutic window in cancers that have lost $\mathrm{p} 53$ function.

Given that most DIPGs contain p53 mutations $(21,22)$, we hypothesize that treatment with ATM inhibitors will selectively radiosensitize $p 53$ mutant tumor cells without affecting the radiosensitivity of adjacent $p 53$ wild-type stromal cells and normal tissue. Consistent with this hypothesis, studies in Atm knockout mice have shown that deletion of both copies of Atm radiosensitizes the intestines and bone marrow but does not radiosensitize other normal tissues, including the brain, skin, lung, and heart $(16,37,38)$. Further supporting the notion of a therapeutic window for treating DIPGs with concurrent radiotherapy and ATM inhibition are 
Table 1. Radiation response of brainstem glioma GEMMs

\begin{tabular}{|c|c|c|c|c|c|c|}
\hline $\begin{array}{l}\text { Mouse } \\
\text { strain }\end{array}$ & p53 & Ink4a/Arf & Atm & Radiosensitivity & $\begin{array}{l}\text { Median } \\
\text { survival } \\
\text { (d) }\end{array}$ & $\begin{array}{l}\text { Median } \\
\text { survival after } \\
\text { radiotherapy } \\
\text { (d) }\end{array}$ \\
\hline$n P A^{f l /+}$ & - & + & + & Resistant & 15 & 42 \\
\hline$n P A^{f l / f l}$ & - & + & - & Sensitive & 16 & 131 \\
\hline$n I A^{f /+}$ & + & - & + & Sensitive & 10 & 122 \\
\hline$n \mid A^{f / f f}$ & + & - & - & Sensitive & 15.5 & 89 \\
\hline$n P I A^{f l /+}$ & - & - & + & Very resistant & 16 & 28 \\
\hline$n P I A^{f l / f l}$ & - & - & - & Resistant & 21.5 & 44 \\
\hline
\end{tabular}

mouse strains were maintained on a mixed genetic background. Male and female littermate controls that retained Atm expression or lacked both alleles of Atm were utilized to minimize the effect of differences in sex, genetic background, and environment on the experimental endpoint.

Primary glioma induction. DF1 chicken fibroblast cells (ATCC) were cultured at $39^{\circ} \mathrm{C}$ in DMEM with high glucose (ATCC) supplemented with 10\% FBS, 2 mM L-glutamine, and 1\% antibiotic-antimycotic. The DF1s were transfected with various RCAS plasmids using X-TremeGENE 9 (Roche). Primary brainstem gliomagenesis was induced by injecting Nestin ${ }^{T V A}$ mice (P3-5) with $1 \times 10^{5}$ virus-producing

findings that Atm-null mice are resistant to radiation-induced cell death in many regions of the central nervous system, including the dentate gyrus, cerebellum, and cerebral cortex (39). Taken together, these results suggest that ATM inhibitors could simultaneously radiosensitize a $p 53$ mutant tumor in the brainstem while protecting the surrounding normal brain tissue. ATM inhibitors that penetrate the blood-brain barrier have been recently developed (19) and are now in clinical trials with concurrent radiotherapy for adults with glioma and brain metastases (NCT03423628); therefore, our findings have important implications for the clinical translation of concurrent ATM inhibition and radiotherapy in children with DIPGs.

Our study showed that $p 53$ wild-type brainstem gliomas respond well to radiotherapy, although they are not further radiosensitized by deletion of Atm. The radiosensitivity of these tumors was mediated by $p 53$ in that deletion of $p 53$ completely attenuated the radiation response. Surprisingly, tumors lacking both $p 53$ and Ink4a/Arf were more resistant to radiation than tumors driven by loss of $p 53$ alone. These findings suggest that $p 53$ and Ink4a/Arf independently regulate tumor response to radiation. Consistent with a recent report highlighting the role of these 2 tumor suppressors in the negative regulation of the NRF2 pathway (29), we found that expression of Nqo1 was significantly increased in tumor cells lacking $p 53$ and Ink4a/Arf. These results support a role for Ink4a/Arf in tumor development outside of its canonical activity as a regulator of $\mathrm{p} 53$ protein stability. In addition to providing a possible mechanism for the heightened radioresistance of $n P I A^{f /+}$ gliomas, increased expression of $\mathrm{Nqo1}$ may also provide a selective pressure in human tumors that acquire mutations in both the $p 53$ and CDKN2A loci (40-43). Interestingly, others have recently reported that substrates for NQO1 that lead to a futile redox cycle using $\mathrm{NAD}(\mathrm{P}) \mathrm{H}$ are effective radiosensitizers (44). Targeting NQO1 during radiotherapy may therefore represent a promising approach to improve outcomes in DIPGs with genotypes that limit radiosensitization by targeting ATM.

\section{Methods}

Mouse strains. All of the mouse strains used in this study have been described previously, including Nestin ${ }^{T V A}, p 53^{f l}, A T M^{f l}$, and Ink4a/Arfl mice (45-48). Nestin ${ }^{T V A}$ mice were provided by Oren Becher (Northwestern University), $p 53^{f}$ mice were provided by Anton Berns (Netherlands Cancer Institute, Amsterdam, the Netherlands), $A T M^{f l}$ mice were provided by Frederick Alt (Boston Children's Hospital, Boston, Massachusetts, USA), and Ink4a/Arfl mice were provided by Ronald DePinho (MD Anderson Cancer Center, Houston, Texas, USA). All
DF1 cells resuspended in $1 \mu \mathrm{L}$. A Hamilton syringe was used to inject the DF1 cells into the brainstem as previously described (34). DF1 cells expressing RCAS-PDGFB, RCAS-Cre, and RCAS-luciferase were injected in equal ratios for all studies except for the FACS experiments. In the FACS experiments, mice were injected with $1 \times 10^{5}$ virus-producing DF1 cells expressing RCAS-PDGFB, RCAS-Cre, RCAS-luciferase, and RCAS-GFP at a 2:2:1:1 ratio.

In vivo imaging of primary gliomas. After chemical hair removal, mice were i.p. injected with $150 \mathrm{mg} / \mathrm{kg}$ D-luciferin (Gold Biotechnology). Mice were anesthetized in a chamber with $2 \%-3 \%$ isoflurane in oxygen, and bioluminescence was assessed 10 minutes after injection of D-luciferin. Mice were monitored biweekly beginning 4.5 weeks after tumor initiation using the IVIS Lumina III (PerkinElmer).

MRI was performed on a subset of mice using a 7 Tesla preclinical scanner (Bruker BioSpec 7/20) equipped with cryogenic receivers (Cryoprobe, Bruker). Mice were anesthetized with isoflurane, and the brain was imaged 3-dimensionally at $0.15 \mathrm{~mm}$ isotropic resolution using a $T 2$-weighted acquisition, with parameters fg-gr and a T1-weighted acquisition. Imaging took place prior to and after injection with a gadolinium-based contrast agent (Magnevist, DOSE) through a tail-vein catheter. The location and size of the brainstem gliomas were determined by manual segmentation.

Radiation treatment. All primary tumor irradiations were performed with the X-RAD 225Cx small animal image-guided irradiator (Precision X-Ray). Mice were placed in a prone position and anesthetized with isoflurane. Fluoroscopy with $40 \mathrm{kVp}, 2.5 \mathrm{~mA}$ x-rays filtered through $2 \mathrm{~mm}$ aluminum was utilized to center the radiation field on the target. Mice were irradiated with $225 \mathrm{kVp}, 13 \mathrm{~mA}$ x-rays filtered through $0.3 \mathrm{~mm}$ copper using a $15 \times 40 \mathrm{~mm}$ rectangular radiation field. The average dose rate was calculated to be $282 \mathrm{cGy} / \mathrm{min}$ using an ionization chamber. Tumor-bearing mice received whole-brain irradiation with right and left lateral fields.

Primary tumor cell lines were irradiated in vitro using either the X-RAD 320 or the X-RAD 160 Biological Irradiator (Precision X-ray Inc.). In the X-RAD 320, cells were treated $50 \mathrm{~cm}$ from the radiation source with $320 \mathrm{kVP}, 10 \mathrm{~mA}$ X-rays, using a $2 \mathrm{~mm}$ aluminum filter. In the X-RAD 160, cells were treated $40 \mathrm{~cm}$ from the radiation source with $160 \mathrm{kVP}, 18 \mathrm{~mA}$ x-rays, using a $2 \mathrm{~mm}$ aluminum filter. Dose rates were calculated by the Radiation Safety Division at Duke University.

Histological analysis. H\&E staining and immunohistochemistry were performed on both frozen and paraffin-embedded sections. Frozen tissues were first fixed in $4 \%$ paraformaldehyde for 24 hours, then transferred to a $30 \%$ sucrose solution for $24-48$ hours, and finally 
A

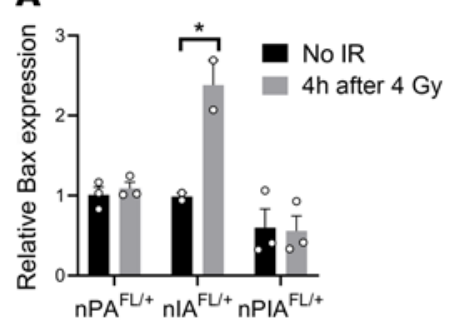

B

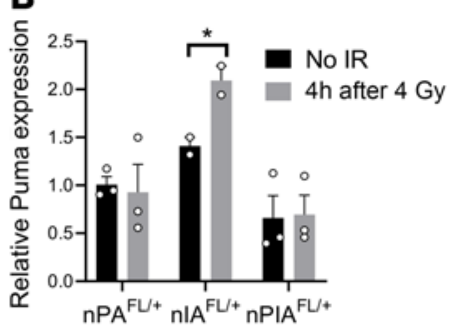

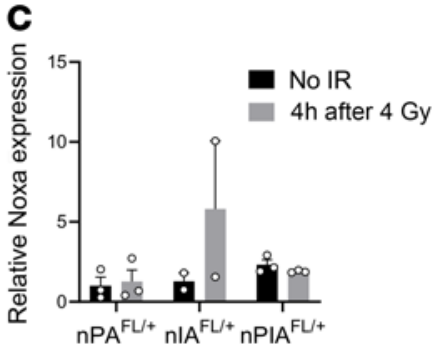

D

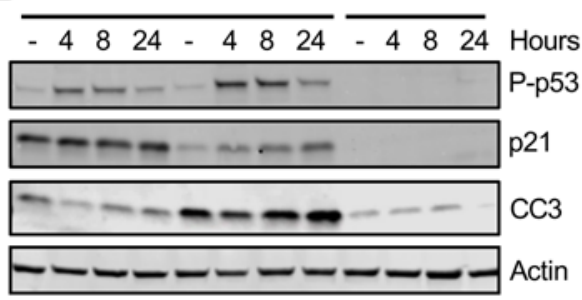

G

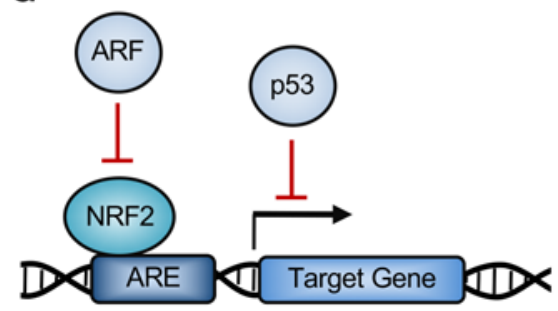

E

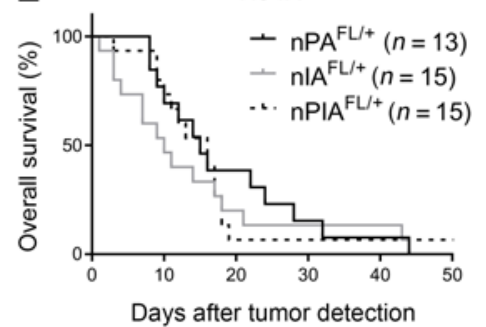

$\mathbf{F}$

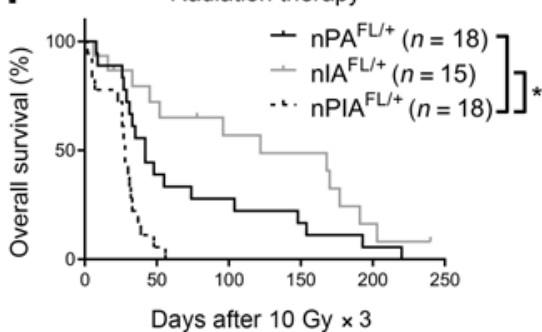

H

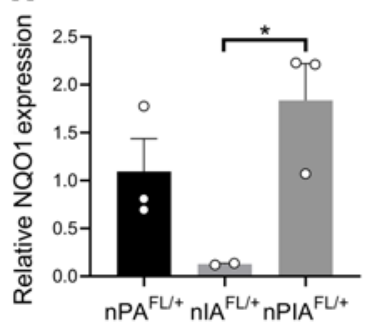

I

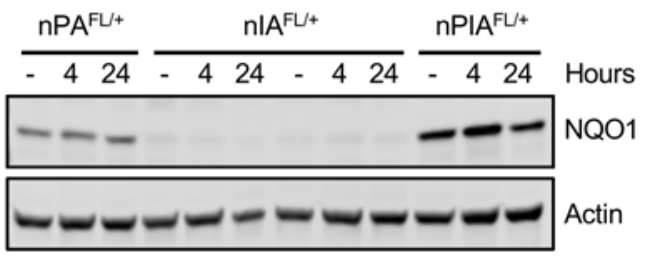

Figure 5. p53 signaling mediates radiosensitivity of Ink4a/Arf-deficient gliomas. Expression of the proapoptotic genes (A) Bax, (B) Puma, and (C) Noxa as quantified by real-time PCR in brainstem glioma cell lines 0 or 4 hours after 4 Gy. ${ }^{*} P<0.05$ by 2-way ANOVA followed by Bonferroni's multiple-comparison test. (D) Western blot showing protein levels of phosphorylated p53 (P-p53), p21, cleaved caspase-3 (CC3), and actin in $n / A^{f / /+}\left(n=2\right.$ cell lines) and $n P I A^{f / /+}$ tumor cell lines at various time points after 4 Gy. Kaplan-Meier plot comparing the survival of $n P A^{f / /+}, n / A^{f l /+}$, and $n P I A^{f l /+}$ mice after (E) tumor detection or (F) treatment with 3 daily fractions of 10 Gy delivered to the whole brain. Control $n P A^{f / /+}$ and $n / A^{f / /+}$ curves were taken from Figures 2 and 3 . ${ }^{*} P<0.05$ by log rank test. (C) Schematic depicting the negative regulation of the NRF2 signaling pathway by ARF and p53. ARF directly binds to NRF2 and prevents its association with the cognate ARE. p53 mediates transrepression of several NRF2 targets, including Nqo1, by binding to ARE-containing promoters. (H) Expression of Nqo1 in primary glioma cell lines as quantified by real-time PCR. ${ }^{*} P<0.05$ by 1-way ANOVA followed by Tukey's multiple-comparison test. (I) Western blot showing NQO1 and actin protein in $n P A^{f / /+}, n I A^{f /+}\left(n=2\right.$ cell lines), and $n P I A^{f /+}$ tumor cell lines after treatment with 4 Gy.

snap-frozen in OCT compound (Sakura Finetek) using a dry ice and isopentane slurry. A cryostat was used to generate $10 \mu \mathrm{m}$ tissue sections. Paraffin-embedded tissues were fixed in 10\% neutralized formalin overnight and preserved in 70\% ethanol until embedding in paraffin. Prior to staining, $5 \mu \mathrm{m}$ tissue sections were deparaffinized in xylene and rehydrated in a graded series of ethanol and water washes.

For immunohistochemistry, endogenous peroxidase activity was blocked by treating sections with $3 \% \mathrm{H}_{2} \mathrm{O}_{2}$, and antigens were retrieved with Antigen Unmasking Solution (Vector Laboratories). Tissues were blocked in $10 \%$ serum with $0.25 \%$ Tween 20 (MilliporeSigma) prior to incubation in primary antibody overnight. The following primary antibodies were used: rabbit polyclonal to HA-probe (1:250, Santa Cruz Biotechnology, sc-805), mouse monoclonal to Ser1981 phosphorylated ATM (1:200, MilliporeSigma, 05-740), rabbit anti-mouse Ser824 phosphorylated Kap1 (1:200, Bethyl Laboratories, A300-767A), and rabbit polyclonal to Ser15 phosphorylated p53 (1:200, Cell Signaling Technology, 9284). The tissues were incubated in biotinylated secondary antibodies for 30 minutes at room temperature and treated with
VECTASTAIN Elite ABC Reagent (Vector Laboratories) per the manufacturer's instructions. The DAB Peroxidase Substrate Kit (Vector Laboratories) was utilized to visualize positive staining prior to counterstaining with Mayer's hematoxylin and dehydrating in a graded series of ethanol and water. Representative images were acquired with a Leica DFC450 bright-field microscope using Leica Suite software. Image (NIH) was utilized to quantify positively stained cells or fractional areas by a single observer blinded to genotype and treatment.

Immunofluorescence. Immunofluorescence was performed on both frozen and paraffin-embedded tissue sections, which were prepared as described in Histological analysis. To perform TUNEL staining, the In Situ Cell Death Detection Kit (Roche) was used per the manufacturer's instructions. Nuclear labeling was performed with VECTASHIELD mounting medium with DAPI (Vector Laboratories). Representative images were acquired with a DFC340 FX fluorescence microscope using Leica Suite software. ImageJ (NIH) was utilized to quantify positively stained cells or fractional areas by a single observer blinded to genotype and treatment. 

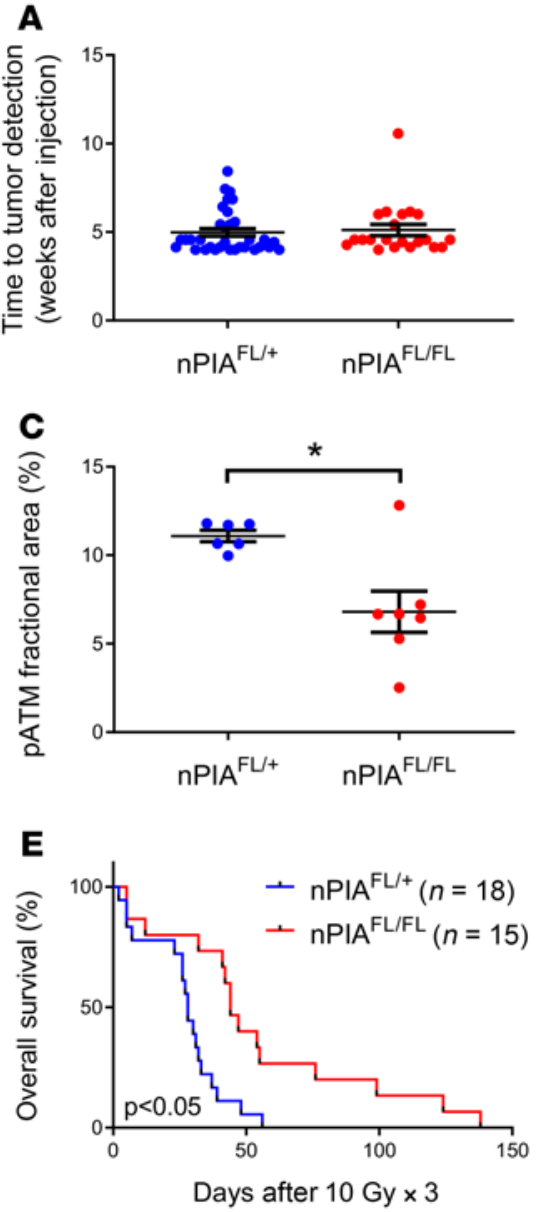
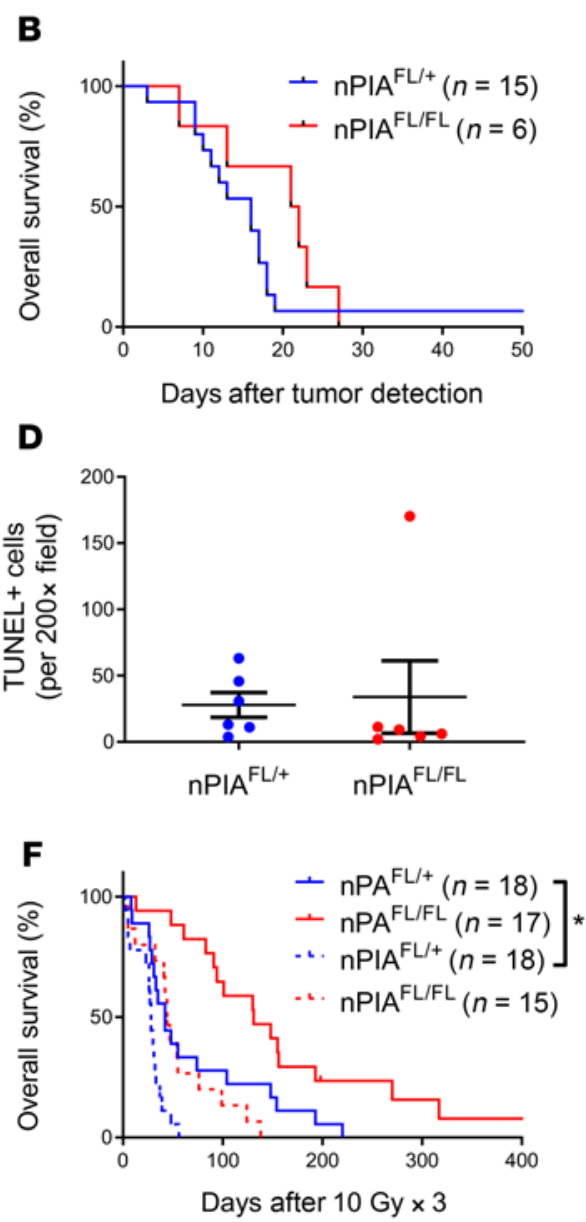

Figure 6. Deletion of Atm improves the radiation response of gliomas driven by loss of $p 53$ and Ink4a/Arf. (A) Time to tumor development after injection in $n P I A^{f / /+}$ and $n P I A^{f / f l}$ mice as detected through in vivo bioluminescence imaging. (B) Kaplan-Meier plot of overall survival in tumor-bearing mice after tumor detection. (C) Quantification of the fractional area of positive staining for PATM 2 hours after 10 Gy in primary brainstem gliomas. (D) Number of TUNEL ${ }^{+}$cells in $n P I A^{f / /+}$ and $n P I A^{f / f f}$ tumors 24 hours after 10 Gy whole-brain irradiation. (E) Kaplan-Meier plot of overall survival in tumor-bearing mice after 3 daily fractions of 10 Gy radiation therapy delivered to the whole brain. $(\mathbf{F})$ Kaplan-Meier plot comparing the survival of mice with $p 53$-deficient gliomas and gliomas lacking both $p 53$ and Ink4a/Arf that were treated with fractionated radiotherapy. Control $n P A^{f / /+}$ and $n P A^{f / / f l}$ curves were taken from Figure $2 .{ }^{*} P<0.05$ by $t$ test ( 2 tailed) when comparing the mean of 2 groups or log rank test when comparing survival curves.
Isolation of primary glioma cell lines. Primary brainstem gliomas were digested at $37^{\circ} \mathrm{C}$ for 15 minutes in dissociation solution containing EBSS, $0.94 \mathrm{mg} / \mathrm{mL}$ papain (Worthington); $0.18 \mathrm{mg} / \mathrm{mL}$ EDTA, $0.18 \mathrm{mg} / \mathrm{mL}$ cysteine; and $0.06 \mathrm{mg} / \mathrm{mL}$ deoxyribonuclease I (DNase). After extensive trituration, the glioma cells were pelleted and resuspended in an ovomucoid solution, containing $0.7 \mathrm{mg} / \mathrm{mL}$ ovomucoid (Worthington) and $0.01 \mathrm{mg} / \mathrm{mL}$ DNase in NeuroCult basal medium (STEMCELL Technologies). The glioma cells were cultured in DMEM with high glucose and pyruvate (Gibco) supplemented with $10 \%$ FBS, 2 mM L-glutamine, and $1 \%$ antibiotic-antimycotic (Gibco). Cells were passaged at least 5 times to enrich for tumor cells and deplete stromal cells.

Clonogenic survival assays. Tumor cells were plated in triplicate at 2 different cell densities and allowed to adhere overnight. After irradiation, cells were incubated 1-2 weeks until colonies were detected in the unirradiated controls. The cells were washed with PBS, fixed with $70 \%$ ethanol, stained with Coomassie Brilliant Blue (Bio-Rad), and rinsed with deionized water. Colonies, defined as collections of at least 50 adjacent cells, were quantified by a single observer to calculate surviving fractions relative to unirradiated controls.

FACS. At the time of tumor initiation, mice were injected with DF1 cells expressing RCAS-GFP, as described in the Primary glioma induction section. Upon detection of a tumor by bioluminescence imaging, primary glioma cells were isolated and red blood cells were lysed using ammonium-chloride-potassium lysing buffer (Lonza). Tumor cells were resuspended in flow buffer (HBSS with $\mathrm{Ca}^{2+}$ and
$\mathrm{Mg}^{2+}, 5 \%$ FBS, 2 mM EDTA) and stained with Zombie Aqua Fixable Viability Kit (BioLegend, 1:200). Living, $\mathrm{GFP}^{+}$glioma cells were flow-sorted using the Astrios Cell Sorter (Beckman Coulter).

Droplet digital PCR to quantify recombination. Genomic DNA was extracted from flow-sorted primary glioma cells using PicoPure DNA extraction kit (Applied Biosystems) and quantified using NanoDrop (Thermo Fisher Scientific). To assess the frequency of Cre-mediated recombination of floxed alleles in the glioma models, we designed hexachloro-fluorescein and fluorescein amidite conjugated TaqMan probes to specifically detect the unrecombined and recombined alleles of $A t m^{f l}, p 53^{f}$, or Ink4a/Arfl. The sequences of primers and probes for these assays were the following: Atm recombined primer, 5'-TCACAACCATCTTCAACCCC-3'; Atm-floxed forward primer, 5'-AATCATCCTTTAATGTGCCTCC-3'; Atm-floxed reverse primer, 5'-TTCATCATCGTCGACCGC-3'; Atm recombined probe, $5^{\prime}$-ACACATGCATGCAGGCAGAGCATCCCT-3'; Atm-floxed probe, 5'-AGCTGTTACTTTTGCGTTTGGTGTGGCG-3'; p53 recombined primer, 5'-TCACCATCACCATGAGACAGG-3'; p53 floxed forward primer, 5'-TGCCCTCCGTCCTTTTTCG-3'; p53 floxed reverse primer, 5'-GGACAGCCAGGACTACACAG-3'; p53 recombined probe, $5^{\prime}$-CTTGATATCGAATTCCTGCAGCCCGGG-3'; p53 floxed probe, 5'-ATGCTATACGAAGTTATCTGCAGCCCGG-3'; Ink4a/Arf recombined primer, 5'-CCTAGAGGTTGATGACAAGG-3'; Ink4a/Arf floxed forward primer, 5'-CTGTGGCAGGATATAACTTCG-3'; Ink4a/Arf floxed reverse primer, 5'-GAATCCCCATCCACTCTGGA-3'; Ink4a/Arf recombined probe, $5^{\prime}$-CATTATACGAAGTTATGGCGCGCCC-3'; and Ink4a/Arffloxed probe, $5^{\prime}$-CTCTGAAAACCTCCAGCGTATTCTGGTA-3'. 
Droplet digital PCR on genomic DNA diluted to $25 \mathrm{ng} / \mu \mathrm{L}$ was performed using the QX200 Droplet Digital PCR System (Bio-Rad) according to the manufacturer's instructions. Suggested PCR cycling conditions were used: $94^{\circ} \mathrm{C}$ for 30 seconds and $60^{\circ} \mathrm{C}$ for 1 minute $(40$ cycles), followed by $98^{\circ} \mathrm{C}$ for 10 minutes. Restriction digestion was performed in the droplet digital PCR reaction using HindIII for the Atm and Ink4a/Arf reactions and CviQI for the $p 53$ reaction. Data were analyzed using the QuantaSoft software (Bio-Rad) by an investigator blinded to genotype. Only samples primarily containing tumor cells (as defined by full recombination of floxed tumor suppressor alleles) were assessed for recombination of the floxed allele of Atm.

Immunoblotting. Primary tumor cells were lysed in cold RIPA buffer (MilliporeSigma) supplemented with Aprotinin (MilliporeSigma), PhosStop tablet (MilliporeSigma), cOmplete mini protease inhibitor cocktail tablet (MilliporeSigma), and 1mM PMSF. Soluble proteins were quantified using the Pierce BCA Protein Assay (Thermo Fisher Scientific) and equal amounts of protein were resolved by SDS-PAGE. Proteins were transferred to nitrocellulose membranes and immunoblotted with specific antibodies for p19ARF (Novus Biologicals, NB200-174, 1:250), p53 (Cell Signaling Technology, 2524, 1:250), Ser15 phosphorylated p53 (Cell Signaling Technology, 9284, 1:250), p21 (Santa Cruz Biotechnology, sc-471, 1:250), cleaved caspase-3 (Cell Signaling Technology, 9661S, 1:1000), NQO1 (Abcam, ab34173, 1:1000), actin (BD Transduction, 612656, 1:10,000) or GAPDH (ProteinTech, 60004-1-IgG, 1:1000). Proteins were visualized using infrared fluorophore-labeled secondary antibodies (Li-Cor Biosciences, IRDye800 and IRDye680) and imaged with the Odyssey imaging system (Li-Cor Biosciences).

Quantitative real-time PCR. Total RNA was extracted from tumor cells using the Direct-zol RNA Kit (Zymo) and reverse transcription was performed using the iScript Advanced cDNA Synthesis Kit (Bio-Rad). Quantitative real-time PCR was utilized to detect mRNA expression using TaqMan Fast Advanced Master Mix (Thermo Fisher Scientific) and TaqMan probes (Thermo Fisher Scientific, Mm00451763_m1 for Noxa, Mm00432051_m1 for Bax, Mm00519268_m1 for Puma, Mm01253561_ m1 for NQO1, Mm99999915_g1 for Gapdh). Gapdh expression was used as an internal control for RNA concentration across samples. Every sample was run in triplicate, and the results were averaged for each assay.
Statistics. All data are presented as mean \pm SEM. Student's $t$ test $(2$ tailed) was utilized to compare the mean of 2 groups. To compare the mean of 3 groups, 1-way ANOVA followed by Tukey's multiple-comparison test were performed. Two-way ANOVA was utilized to examine the interaction between genotype and radiation or time point followed by Bonferroni's multiple-comparison test for pairwise comparisons. Kaplan-Meier analysis was performed for the survival studies, followed by the log rank test for statistical significance. A $P$ value less than 0.05 indicated significance. Prism 7 (GraphPad Software Inc.) was used for the statistical analysis.

Study approval. All animal studies were approved by the IACUC at Duke University.

\section{Author contributions}

$\mathrm{KD}$, OJB, and DGK conceived the study. KD, MC, OJB, and DGK designed experiments. KD, BFS, JSM, JB, and MC generated the data. DMC provided mentorship and oversaw the generation of primary brainstem gliomas. KD and DGK analyzed the data. NTW irradiated the mice. LL tattooed and genotyped the mice. YM performed the histology. KD and DGK wrote the manuscript. $\mathrm{KD}, \mathrm{MC}, \mathrm{OJB}$, and DGK reviewed the manuscript.

\section{Acknowledgments}

This work is dedicated to the memory of Rose Sugarman, who died from a DIPG. We thank John Nouls from the Duke Center for In Vivo Microscopy for assistance in obtaining the MRI. This work was supported by grants to DGK from the National Cancer Institute (R35 CA197616), Pediatric Brain Tumor Foundation, Hannah's Heroes St. Baldrick's Research Grant, and The Leon Levine Foundation. DGK and KD were supported by the National Cancer Institute (Duke Brain SPORE P50-CA19099). MC was supported by the National Cancer Institute (F30 CA206424). OJB was supported by Maddox's Warriors, the Fly A Kite Foundation, Cristian Rivera Foundation, and the Rory David Deutsch Foundation.

Address correspondence to: David G. Kirsch, Duke University Medical Center, DUMC Box 91006, Durham, North Carolina 27708, USA. Phone: 919.681.8605; Email: david.kirsch@duke.edu.
1. Hargrave D, Bartels U, Bouffet E. Diffuse brainstem glioma in children: critical review of clinical trials. Lancet Oncol. 2006;7(3):241-248.

2. Jansen MH, van Vuurden DG, Vandertop WP, Kaspers GJ. Diffuse intrinsic pontine gliomas: a systematic update on clinical trials and biology. Cancer Treat Rev. 2012;38(1):27-35.

3. Ward JF. Some biochemical consequences of the spatial distribution of ionizing radiation-produced free radicals. Radiat Res. 1981;86(2):185-195.

4. Moding EJ, Kastan MB, Kirsch DG. Strategies for optimizing the response of cancer and normal tissues to radiation. Nat Rev Drug Discov. 2013;12(7):526-542.

5. Werbrouck C, et al. TP53 pathway alterations drive radioresistance in diffuse intrinsic pontine gliomas (DIPG). Clin Cancer Res. 2019;25(22):6788-6800.

6. Lee JH, Paull TT. ATM activation by DNA double-strand breaks through the Mre11-Rad50Nbs1 complex. Science. 2005;308(5721):551-554.

7. Bakkenist CJ, Kastan MB. DNA damage activates
ATM through intermolecular autophosphorylation and dimer dissociation. Nature. 2003;421(6922):499-506.

8. Kozlov S, Gueven N, Keating K, Ramsay J, Lavin MF. ATP activates ataxia-telangiectasia mutated (ATM) in vitro. Importance of autophosphorylation. J Biol Chem. 2003;278(11):9309-9317.

9. Shiloh Y, Ziv Y. The ATM protein kinase: regulating the cellular response to genotoxic stress, and more. Nat Rev Mol Cell Biol. 2013;14(4):197-210.

10. Chaturvedi P, et al. Mammalian Chk2 is a downstream effector of the ATM-dependent DNA damage checkpoint pathway. Oncogene. 1999;18(28):4047-4054.

11. Matsuoka S, Rotman G, Ogawa A, Shiloh Y, Tamai K, Elledge SJ. Ataxia telangiectasia-mutated phosphorylates Chk2 in vivo and in vitro. Proc Natl Acad Sci U S A . 2000;97(19):10389-10394.

12. Matsuoka S, Huang M, Elledge SJ. Linkage of ATM to cell cycle regulation by the Chk2 protein kinase. Science. 1998;282(5395):1893-1897.

13. White D, et al. The ATM substrate KAP1 controls
DNA repair in heterochromatin: regulation by HP1 proteins and serine 473/824 phosphorylation. Mol Cancer Res. 2012;10(3):401-414.

14. Paterson MC, Smith PJ. Ataxia telangiectasia: an inherited human disorder involving hypersensitivity to ionizing radiation and related DNA-damaging chemicals. Annu Rev Genet. 1979;13:291-318.

15. Gotoff SP, Amirmokri E, Liebner EJ. Ataxia telangiectasia. Neoplasia, untoward response to $\mathrm{x}$-irradiation, and tuberous sclerosis. Am J Dis Child. 1967;114(6):617-625.

16. Barlow C, et al. Atm-deficient mice: a paradigm of ataxia telangiectasia. Cell. 1996;86(1):159-171.

17. Rainey MD, Charlton ME, Stanton RV, Kastan MB. Transient inhibition of ATM kinase is sufficient to enhance cellular sensitivity to ionizing radiation. Cancer Res. 2008;68(18):7466-7474.

18. Hickson I, et al. Identification and characterization of a novel and specific inhibitor of the ataxia-telangiectasia mutated kinase ATM. Cancer Res. 2004;64(24):9152-9159.

19. Durant ST, et al. The brain-penetrant clinical 
ATM inhibitor AZD1390 radiosensitizes and improves survival of preclinical brain tumor models. Sci Adv. 2018;4(6):eaat1719.

20. Barton KL, et al. PD-0332991, a CDK4/6 inhibitor, significantly prolongs survival in a genetically engineered mouse model of brainstem glioma. PLoS One. 2013;8(10):e77639.

21. Wu G, et al. The genomic landscape of diffuse intrinsic pontine glioma and pediatric non-brainstem high-grade glioma. Nat Genet. 2014;46(5):444-450.

22. Khuong-Quang DA, et al. K27M mutation in histone H3.3 defines clinically and biologically distinct subgroups of pediatric diffuse intrinsic pontine gliomas. Acta Neuropathol. 2012;124(3):439-447.

23. Liu X, et al. Self-inflicted DNA double-strand breaks sustain tumorigenicity and stemness of cancer cells. Cell Res. 2017;27(6):764-783.

24. Biddlestone-Thorpe L, et al. ATM kinase inhibition preferentially sensitizes p53-mutant glioma to ionizing radiation. Clin Cancer Res. 2013;19(12):3189-3200.

25. Cordero FJ, et al. Histone H3.3K27M represses p16 to accelerate gliomagenesis in a murine model of DIPG. Mol Cancer Res. 2017;15(9):1243-1254.

26. Jeong $Y$, et al. Role of KEAP1/NRF2 and TP53 mutations in lung squamous cell carcinoma development and radiation resistance. Cancer Discov. 2017;7(1):86-101.

27. Wang T, Hu P, Li B, Zhang JP, Cheng YF, Liang YM. Role of Nrf2 signaling pathway in the radiation tolerance of patients with head and neck squamous cell carcinoma: an in vivo and in vitro study. Onco Targets Ther. 2017;10:1809-1819.

28. Singh A, Bodas M, Wakabayashi N, Bunz F, Biswal S. Gain of Nrf2 function in non-small-cell lung cancer cells confers radioresistance. Antioxid Redox Signal. 2010;13(11):1627-1637.

29. Chen D, et al. NRF2 is a major target of ARF in p53-independent tumor suppression. Mol Cell. 2017;68(1):224-232.e4.

30. Faraonio R, et al. p53 suppresses the Nrf2-dependent transcription of antioxidant response genes. JBiol Chem. 2006;281(52):39776-39784.

31. Tung MC, et al. Mutant p 53 confers chemoresistance in non-small cell lung cancer by upregulating Nrf2. Oncotarget. 2015;6(39):41692-41705

32. Tang L, et al. Role of metabolism in cancer cell radioresistance and radiosensitization methods. JExp Clin Cancer Res. 2018;37(1):87.

33. Castle KD, Chen M, Wisdom AJ, Kirsch DG. Genetically engineered mouse models for studying radiation biology. Transl Cancer Res. 2017;6(Suppl 5):S900-S913.

34. Becher OJ, et al. Preclinical evaluation of radiation and perifosine in a genetically and histologically accurate model of brainstem glioma. Cancer Res. 2010;70(6):2548-2557.

35. Powell SN, et al. Differential sensitivity of p53(-) and p53(+) cells to caffeine-induced radiosensitization and override of G2 delay. Cancer Res. 1995;55(8):1643-1648.

36. Moding EJ, et al. Atm deletion with dual recombinase technology preferentially radiosensitizes tumor endothelium. J Clin Invest. 2014;124(8):3325-3338.

37. Westphal CH, Rowan S, Schmaltz C, Elson A, Fisher DE, Leder P. atm and $\mathrm{p} 53$ cooperate in apoptosis and suppression of tumorigenesis, but not in resistance to acute radiation toxicity. Nat Genet. 1997;16(4):397-401.

38. Ch'ang HJ, et al. ATM regulates target switching to escalating doses of radiation in the intestines. Nat Med. 2005;11(5):484-490.

39. Herzog KH, Chong MJ, Kapsetaki M, Morgan JI, McKinnon PJ. Requirement for Atm in ionizing radiation-induced cell death in the developing central nervous system. Science. 1998;280(5366):1089-1091.
40. Cancer Genome Atlas Research Network. Comprehensive genomic characterization of squamous cell lung cancers. Nature. 2012;489(7417):519-525.

41. Smeds J, Berggren P, Ma X, Xu Z, Hemminki K, Kumar R. Genetic status of cell cycle regulators in squamous cell carcinoma of the oesophagus: the CDKN2A (p16(INK4a) and p14(ARF)) and p53 genes are major targets for inactivation. Carcinogenesis. 2002;23(4):645-655.

42. Sanchez-Cespedes $M$, et al. Inactivation of the INK4A/ARF locus frequently coexists with TP53 mutations in non-small cell lung cancer. Oncogene. 1999;18(43):5843-5849.

43. Soufir N, et al. Association between INK4a-ARF and p53 mutations in skin carcinomas of xeroderma pigmentosum patients. J Natl Cancer Inst. 2000;92(22):1841-1847.

44. Motea EA, et al. NQO1-dependent, tumor-selective radiosensitization of non-small cell lung cancers. Clin Cancer Res. 2019;25(8):2601-2609.

45. Jonkers J, Meuwissen R, van der Gulden $H$, Peterse H, van der Valk M, Berns A. Synergistic tumor suppressor activity of BRCA2 and p53 in a conditional mouse model for breast cancer. $\mathrm{Nat}$ Genet. 2001;29(4):418-425.

46. Zha S, et al. Ataxia telangiectasia-mutated protein and DNA-dependent protein kinase have complementary $\mathrm{V}(\mathrm{D})$ J recombination functions. Proc Natl Acad Sci U S A. 2011;108(5):2028-2033.

47. Holland EC, Hively WP, Gallo V, Varmus HE. Modeling mutations in the G1 arrest pathway in human gliomas: overexpression of CDK4 but not loss of INK4a-ARF induces hyperploidy in cultured mouse astrocytes. Genes Dev. 1998;12(23):3644-3649.

48. Aguirre AJ, et al. Activated Kras and Ink4a/ Arf deficiency cooperate to produce metastatic pancreatic ductal adenocarcinoma. Genes Dev. 2003;17(24):3112-3126. 